\title{
Wer war Pseudo-Dionysios?
}

Den Kreis der Möglichkeiten für die Beantwortung dieser Frage haben neuere Arbeiten wesentlich verkleinert. Besonders durch Stiglmayr ${ }^{1}$ ) ist erwiesen worden, dafs die Pseudo-Dionysischen Schriften nicht lange vor dem Jahre 500, höchst wahrscheinlich nach dem Henotikon Kaiser Zenos, d. h. nach 482, und zwar in Syrien geschrieben sein müssen. Sollte es nicht möglich sein, sie noch näher zu lokalisieren und vielleicht gar den grofsen Unbekannten zu entdecken, der sie geschrieben hat? Bei der Beschäftigung mit der sog. Kirchengeschichte des Zacharias Rhetor (Historia miscella ed. Land, Anecdota Syriaca III), die Herr Karl Ahrens in Ploen und ich demnächst deutsch zu veröffentlichen gedenken, hat sich mir eine Möglichkeit aufgedrängt, die ich nicht unausgesprochen lassen möchte, auf die Gefahr hin, dafs man wir vorwirft, das Gras wachsen zu hören.

Nach den bisherigen Untersuchungen dürfte feststehen, dafs die dionysischen Schriften in den Kreisen der Severianer zuerst bekannt und benutzt worden sind. Severos selbst verwertet die Schrift von den göttlichen Namen und den vierten Brief an Gaios (Stiglm. 49). Noch auf dem Religionsgespräch zu Konstantinopel im Jahre 533 haben sich die Severianer auf Dionysios berufen ${ }^{2}$ ), obschon gleichzeitig der

1) Da ich hier nur eine Notiz zu geben gedenke, so darf ich mir die Anführung der einschlägigen Litteratur, soweit sie nicht direkt zu dem von mir neu in die Debatte geworfenen Moment gehört, wohl ersparen. Einen trefflichen Überblick über den Stand der Dionysiosfrage hat jüngst Bonwetsch in der Realencyklopädie f. prot. Theol. u. K. Bd 4, 687-696 gegeben. Unter der im Text mehrfach angeführten Abhandlung von Stiglmayr ist die Arbeit über „Das Aufkommen der Pseudo-Dionysischen Schriften und ihr Eindringen in die christliche Litteratur bis zum Laterankonzil 649" im 4. Jahresbericht des offentlichen Privatgymnasiums an der Stella matutina zu Feldkirch $1895 \mathrm{zu}$ verstehen.

2) Mansi 8, 820. In der Historia mıscella 9, 15 ist die dénous erhalten, welche die Severianer dem Kaiser vor dem Gespräche eingereicht hatten (Mansi 8, 818: nos satisfactionis chartulam de fide nostra compositam piissimo imperatori porreximus et in ea omnia, quae nobis ambigua videbantur et scandalizabant nos, intexuimus). In dieser Bittschrift ist das Zitat aus Pseudo-Dionysios enthalten, und zwar ist es aus De div. nom. 1, 4 (Migne, S. G. 3,592 A) entnommen. Die Stelle 
Patriarch Ephräm von Antiochien (527-545) eine Stelle aus Dionysios im orthodoxen Sinne gegen die Häretiker auslegt (Stiglm. 51) und Leontios von Byzanz wie Joannes von Skythopolis die Schriften für echt und orthodox hielten. Ohne Zweifel erinnert „die ganze Haltung der Dionysischen Schriften in der Christologie an die im Henotikon Kaiser Zenons" (Bonwetsch 691, 15 nach Stiglmayr). St. 40 hat sogar die Frage aufgeworfen, wenn er sich auch nicht getraut, sie zu bejahen, ob nicht Dionysios direkt die Tendenz verfolgte, das Henotikon zu empfehlen und seine Einführung zu unterstützen.

In dem von Zacharias Rhetor verfafsten „Leben des zu den Heiligen gehörigen Vaters Isaias des Asketen", das wir im Anhang zu unserer Übersetzung der Kirchengeschichte, gleichfalls nach Land, veröffentlichen werden ${ }^{1}$ ), wird ein Scholastikos aus Gaza, Namens Dionysios, genannt, von dem der Verfasser erzählt, dafs er sich bei Isaias geistlichen Rat geholt habe. Es ist derselbe Dionysios, dessen auch der anonyme Verfasser der Lebensbeschreibung Petros' des Iberers (ed. Raabe, Leipzig 1895, S. 95 f.) gedenkt. Dieser nennt ihn einen „Rechtgläubigen“, der auch eine rechtgläubige Mutter und Grolsmutter und

lautet in Ahrens' thersetzung: „Jener Dionysios vom Areiospagos, der aus dem Dunkel und Irrtume des Heidentums (vgl. hierzu cael. hier. 9, 3 p. $361 \mathrm{~A}$ ) zum vorzüglichen Lichte der Erkenntnis Gottes durch unseren Führer Paulos gelangte, sagte in dem Buche, das er über die göttlichen Namen der Dreifaltigkeit verfafste: Indem wir sie als menschenliebend finden, sagen wir, dafs sie menschenliebend ist, wie es sich gebührt, da sie mit dem Unseren in Wahrheit in einer ihrer Personen vollkommene Gemeinschaft hat, indem sie die Niedrigkeit unserer Menschheit zu sich heran- und hinaufzog, aus der auf unausprechliche Weise der einfache Jesus zusammengesetzt wurde, und indem der ein zeitliches Dasein annahm, der von Ewigkeit an und oberhalb aller Zeiten war, und der ohne Veränderung und Verwirrung von einer uns ähnlichen Natur ward, der hoch erhaben war über alle Ordnungen und Naturen." Ich bemerke beiläufig, dafs der Verfasser der Historia miscella oder seine Quelle die Rückberufung der Bischöfe nach dem Nikaaufstand anzusetzen scheint, was gegen die von Loofs, Leontius von Byzanz, 283*) bevorzugte Datierung des Religionsgespräches auf 531 und für die gewöhnliche Ansetzung sprechen dürfte.

1) Dieser Isaias ist identisch mit dem Mönche, von dem in Migne S. G. 40,

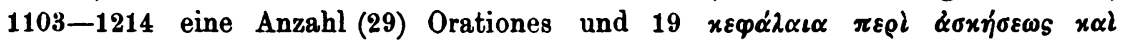
jंov $\chi^{i} \alpha s$ gedruckt sind. Man hat den Verfasser dieser Schriften mit dem von Rufin, Histor. Monach. 12 (ed. Preuschen $63 \mathrm{f}$.) und von Palladius, Hist. Laus. 55-58 (Migne S. G. 34, 1138) erwähnten Isaias identifizieren wollen. Nun ist die 25. Oratio des Isaias ad Petrum abbatem discipulum suum gerichtet, und eben ein solcher Abt Petros wird in der Vita als intimer Schüler des Isaias zweimal erwähnt. Unser Isaias starb zwischen 385-390, wenige Monate vor Petros dem Iberer. Sehr wahrscheinlich sind ihm auch die Apophthegmata zuzuschreiben, die Cotelerius im ersten Bande seiner Eccl. graec. monumenta p. 445 f. und 596 abgedruckt hat. 
eben solche Brüder - ein auserwähltes und gesegnetes Geschlecht hatte und von Glauben und von Liebe zum Heiligen glühte. Er erzählt, dafs er mit Petros in lebhaftem Verkehr gestanden und sich dabei auch das leibliche Wohl des Seligen habe angelegen sein lassen, indem er für seine bequeme Unterkunft in einem Dorfe bei Gaza sorgte. Dionysios mufs nach dem Anonymus ein wohlhabender Mann gewesen sein, dem es nicht darauf ankam, dreihundert Dareiken auf den Bau und die Herrichtung der Stätte für Petros auszugeben; freilich nahm er sie, wie er selbst erzählt (S. 96), wenige Tage später von einer Seite wieder ein, von welcher er es niemals erwartet hätte. Eine gute Illustration zu dem schönen Worte: Wohlthun trägt Zinsen.

Nun ist zu bemerken, dafs der Kreis von Frommen, der in den beiden genannten Lebensbeschreibungen und, fast noch deutlicher hervortretend, in der Vita des Severos, die gleichfalls Zacharias Rhetor verfafste (von Spanuth 1893, leider nur syrisch, herausgegeben), eine Rolle spielt, zwar streng antichalkedonensisch denkt, aber mindestens ebenso antieutychianisch. ${ }^{1}$ ) Die „Richtung“ dieser Männer entspricht genau derjenigen, welche dem Henotikon zu Grunde liegt, und Kaiser Zenon gilt ihnen als die "Vollendung der Gottesfurcht".

Die Dionysischen Schriften sind gerade in Palästina zuerst auch von Orthodoxen benutzt worden (vgl. Loofs a. a. 0. 269). Sie in Gaza zu lokalisieren, hat sehr viel Verlockendes. Grade hier lälst sich ein solcher Versuch, neuplatonische Ideen in den Dienst christlicher Spekulation zu stellen (Stiglm. 43), trefflich begreifen. Hier schrieb Prokopios (465-528) seine Widerlegung des Proklos. Der Verfasser unserer Schriften ist als Heide geboren (cael. hier. 9, 3, s. o. S. 302 Anm. 2) und mit hellenischer Weisheit wohl vertraut (ep. 7, 2 p. 1080). Die Vita des Severos zeigt uns, wie zahlreiche Utbertritte grade aus den gebildeten Kreisen damals in Gaza stattgefunden haben müssen.

Aber warum mufs grade Dionysios, der Scholastikos, der Verfasser sein? Das weifs ich natürlich auch nicht; denn mir steht nicht mehr Material zur Verfügung, als ich im Vorstehenden gegeben habe. Indessen wolle man bedenken: es ist wenigstens nicht unmöglich, dafs der Verfasser den Namen geführt hat, den er sich beilegte. Zwar ist mir der pseudepigraphe Charakter seiner Schriftstellerei ganz aufser Frage, und ich möchte meine bescheidene Anregung nicht mit all den

1) Hierfür wird unsere thbersetzung der Historia miscella zahlreiche Belege bringen. Besonders deutlich tritt der antieutychianische Standpunkt auch in den "Plerophorien" des Joannes von Gaza hervor, die F. Nau jüngst in der Revue de l'Orient chrétien $(3,1898,232-259.337-392)$ in französischer t'bersetzung veröffentlicht hat. 
Schwierigkeiten beladen, durch die früher die Hypothese „Dionysios von Rhinokolura" bedrückt wurde. Aber warum verfiel der Verfasser just auf Dionysios den Areopagiten, dessen nur aus einer Schriftstelle bekannter Name doch nicht ohne weiteres verführerisch wirken mochte? Es ist doch zum mindesten ein auffälliges Zusammentreffen, dafs sich uns zu einer Zeit und'in einem Orte, wo wir ihn brauchen können, ein Dionysios vorstellt, der nach seinem Berufe und nach der Schilderung, die wir von ihm erhalten, recht wohl die Schriften verfafst haben kann, nach deren Autor man durch die Jahrhunderte vergeblich gesucht hat.

Und seine Freunde und Bekannten? Vorab Severos selbst? Die müssen doch, so kann man mit Grund fragen, gewufst haben, dals dieser Mann die Schriften schrieb, die unter dem Namen des alten Atheners solches Aufsehen zu machen bestimmt waren? Auch darauf weifs ich keine genügende Antwort. Aber weifs denn überhaupt jemand diese Frage zu beantworten, die doch, ganz abgesehen von der speziellen Anwendung auf den Mann Dionysios, sich jedem aufdrängen mufs? Wie war es möglich, dafs zwischen $482-500$ von einem in den engeren Kreisen seiner Gesinnungsgenossen doch jedenfalls sehr bekannten Autor Schriften geschrieben wurden, die man ein Menschenalter später ohne Gefahr sofortiger Entlarvung trotz energischen Widerspruches (Hypatios!) als urchristlich ausgeben konnte? Ist das überhaupt möglich gewesen, so darf man dieses Bedenken gegen meinen Dionysios nicht ins Feld führen.

Giefsen.

G. Krüger. 